\title{
Avaliação em Massa de Imóveis Rurais Através da Regressão Clássica e da Geoestatística
}

\author{
Mass Evaluation of Rural Buildings Through Classical Regression and \\ Geostatistics
}

\author{
Lais Thuany Cardoso Theodoro ${ }^{1}$ \\ Marlene Salete Uberti ${ }^{2}$ \\ Mauro Antonio Homem Antunes ${ }^{3}$ \\ Paula Debiasi 4
}

Recebido em março de 2019.

Aprovado em abril de 2019.

\begin{abstract}
RESUMO
A concepção do Imposto Territorial Rural - ITR está enraizada em métodos subjetivos que tornam esse tributo ineficiente e incoerente com seus propósitos originais. Visando fundamentar a base tributável do imposto e tornar o processo de cálculo mais transparente, este trabalho teve por objetivo verificar se a avaliação em massa de imóveis rurais pode ser utilizada como base para o cálculo do ITR. Baseando-se nas metodologias avaliatórias preconizadas pela NBR 14.653:3, utilizando os dados e mapas do Projeto de Irrigação e Drenagem da Cana-de-Açúcar - PROJIR na Região Norte Fluminense - RJ, aplicou-se a regressão múltipla em conjunto com a geoestatística na elaboração da Planta de Valores Genéricos - PVG em uma área rural. Para analisar o desempenho da avaliação foram utilizadas métricas recomendadas pela International Association of Assessing Officers - IAAO, as quais indicaram que, na regressão múltipla, o valor obtido da mediana das razões de avaliação $(0,98)$ enquadrou-se no intervalo estabelecido pela norma $(0,90$ a 1,10$)$, enquanto que o Coeficiente de Dispersão - COD (24,60\%) e o Price Related Differential - PRD (1,23) mostraram-se superiores aos estabelecidos (20\% e 1,03, respectivamente). Isto indica que, para a região do PROJIR, seria necessária uma quantidade maior de amostras para alcançar os intervalos estipulados pela norma. De acordo com os resultados obtidos nos testes estatísticos da regressão múltipla, e a dependência espacial comprovada pela análise dos
\end{abstract}

\footnotetext{
${ }^{1}$ Universidade Federal Rural do Rio de Janeiro. Instituto de Tecnologia, Departamento de Engenharia, Brasil. E-mail: lais_thuany@hotmail.com

2Universidade Federal Rural do Rio de Janeiro. Instituto de Tecnologia, Departamento de Engenharia, Brasil. E-mail: msuberti @ufrrj.br

${ }^{3}$ Universidade Federal Rural do Rio de Janeiro. Instituto de Tecnologia, Departamento de Engenharia, Brasil. E-mail: mauroantunes@ufrrj.br

${ }^{4}$ Universidade Federal Rural do Rio de Janeiro. Instituto de Tecnologia, Departamento de Engenharia, Brasil. E-mail: paula @ufrrj.br
} 
semivariogramas, conclui-se que a utilização da regressão múltipla em conjunto com a krigagem ordinária, na avaliação em massa de imóveis rurais, é viável para fins de impostos e outras aplicações.

PALAVRAS-CHAVE: Planta de valores. Econometria. Krigagem Ordinária.

\begin{abstract}
The concept of Rural Territorial Tax (ITR) is rooted in subjective methods that turn this tribute inefficient and inconsistent with their original purposes. Thus, this work had the objective to evaluate the hypotheses that the mass evaluation of rural land can be efficiently used to obtain the ITR, aiming at a more transparent process of taxation with a plausible taxable base. Evaluation methodologies preconized by the Brazilian Normalization NBR 14.653:3 (ABNT, 2004) were applied to data and maps using multiple regression along with geostatistics to generate the standard ground value of rural land. The study area was the Sugarcane Irrigation and Drainage Project-PROJIR, located at the Northern of Rio de Janeiro State, Brazil. The metrics recommended by the International Association of Assessing Officers-IAAO (2013) were used to analyze the performance of land price evaluation. The results indicated that for the multiple regression, the median of the evaluation ratios (0.98) was within the established interval (0.90 a 1.10), while the coefficient of dispersion-COD was $24.60 \%$ and the Price Related Differential-PRD was 1.23 , which were higher than the recommended values (20\% and 1.03, respectively). This indicates that, for the PROJIR region, would be necessary a higher quantity of samples to reach the intervals stipulated by the standards. According to the results obtained in the statics tests of multiple regression, and the space dependency by the semivariograms analysis, this concludes the use of multiple regression in conjunction with the ordinary kriging, in the mass evaluation of rural properties, is viable for purposes of taxes and other applications.
\end{abstract}

KEYWORDS: Plant of values. Econometrics. Ordinary Kriging.

\title{
Introdução
}

O Imposto Territorial Rural (ITR) foi instituído no Brasil com a finalidade de atenuar problemas como a concentração de terras, a subutilização das mesmas e gerar receita, além de permitir uma maior fiscalização sobre as propriedades rurais. De acordo com a constituição Federal, artigo 153 (Brasil, 1988), o ITR deveria servir como um mecanismo para proporcionar uma mudança no uso e propriedade da terra; porém, devido à legislação que regulamenta tal imposto e aos aspectos políticos que 
influenciam as decisões administrativas sobre ele, o mesmo torna-se ineficiente, deixando de cumprir o fim a que se destina.

A Lei Federal n 9.393/96 (Brasil, 1996), que regulamenta o ITR, impôs como uma de suas principais características a auto declaração dos valores da terra e de suas benfeitorias. E, como não há um pleno controle, esses valores ficam sujeitos a imprecisões, o que pode ocasionar desigualdade entre as propriedades que são tributadas de tal imposto.

Segundo a Secretaria da Receita Federal - SRF (BRASIL, 2012), agente que arrecada atualmente o imposto, a arrecadação do ITR no ano de 2011 foi de aproximadamente $\mathrm{R} \$ 603$ milhões e no ano de 2012 foi de $\mathrm{R} \$ 677$ milhões. Utilizando-se a área estimada pelo Instituto Nacional de Colonização e Reforma Agrária - INCRA em 2012, de 605 milhões de ha, e a arrecadação do imposto no mesmo ano, tem-se um valor de $1,12 \mathrm{R} \$ /$ ha.

Comparando a arrecadação do ITR em relação ao PIB, do Brasil com a dos países desenvolvidos, para o período de 2002 a 2005, temos: Estados Unidos: 3,09\%; Canadá: 3,05\%; Argentina: 0,82\%; Brasil: 0,46\%. O que mostra o atraso do Brasil frente aos países desenvolvidos em relação ao tema da valoração cadastral rural, cujo valor do ITR é irrisório e não cumpre com seu papel regulador (OLIVEIRA, 2010).

Uma superfície de valores, gerada através de um modelo que contém variáveis que representam fielmente o mercado imobiliário, pode ser utilizada como base de cálculo para a cobrança dos tributos imobiliários, garantindo assim a equidade fiscal e a prática da justiça tributária (LIPORONI, 2007). Mas ainda assim, a maioria dos estudos de avaliação em massa é focado em áreas urbanas, sendo utilizados métodos declaratórios em áreas rurais.

Somente com a implantação do Cadastro Técnico Rural e a adequação dos mecanismos de cálculo e cobrança, o ITR poderá se tornar um instrumento de gestão do espaço rural e de regularização fundiária, diminuindo a evasão de receita e aumentando a justiça fiscal na tributação (UBERTI, 2016).

A maior mudança no ITR ocorreu através da Lei 11.250/2005, que permite atribuir aos municípios conveniados a fiscalização, o lançamento e a 
arrecadação integral da SRF (REYDON e OLIVEIRA, 2012). Contudo, apenas poucos municípios aceitaram, pois não há uma metodologia de avaliação de imóveis rurais passível de ser utilizada como base para a obtenção do ITR.

Sendo assim, propõe-se nesse trabalho a avaliação em massa de imóveis rurais com uso de regressão múltipla em conjunto com a geoestatística, através de metodologias avaliatórias preconizadas pela NBR 14.653:3 - Avaliação de Bens: Imóveis Rurais (ABNT, 2004), com a finalidade de que tal avaliação possa ser utilizada de maneira eficiente para gerar uma superfície de valores que possa ser utilizada para fundamentar a base de cálculo do ITR.

\section{Imposto Territorial Rural (ITR)}

O ITR é um tributo de apuração anual direcionado a pessoas que tenham propriedade, domínio útil ou posse de imóvel localizado fora da zona urbana do município (LEI nº 9.393 de 1996).

A responsabilidade do ITR passou por três fases, a primeira que é desde sua criação até 1979, quando o mesmo era de responsabilidade do Instituto Nacional de Colonização e Reforma Agrária (INCRA), a segunda de 1980 até 1990, quando a legislação passa por significativas modificações, e a terceira que começa em 1990 quando a responsabilidade do tributo passa do INCRA para a Receita Federal. Em seguida a mesma é passada para as esferas Estadual, Municipal e então retornou a Federal.

O objetivo do ITR segundo a Constituição Federal, artigo 153 (Brasil, 1988), é servir como um instrumento para viabilizar uma mudança no uso e posse da terra, porém, de acordo com Neto (1992), os propósitos de desestímulo à subutilização da terra, geração de receita e instrumento distributivista do ITR, desde sua implantação, na constituição de 1891 até 1992 não foram cumpridos. E, até o presente momento, ainda não são.

De acordo com os autores Vilarinho (1989), Neto (1992) e Oliveira (2010), há uma grande evasão de receita na cobrança do ITR e os principais 
motivos para tal evasão são: que tanto o Valor da Terra Nua quanto o grau de utilização do imóvel rural, que são a base de cálculo do ITR, são fornecidos de forma declaratória pelo proprietário do imóvel, além da falta de fiscalização por parte dos responsáveis na cobrança do tributo. O que faculta a declaração de valores não condizentes com a realidade, fazendo com que as terras sejam tributadas segundo o interesse de seus proprietários.

A implementação das leis mais atuais sobre a cobrança do ITR, $10.267 / 2001$ e $11.250 / 2005$, não alteraram os principais problemas relacionados à cobrança desse tributo: sua auto-declaração (subdeclarada) e fiscalização precária, prosseguindo assim a grande evasão de receita (OLIVEIRA, 2010).

\section{Geoestatística}

Para trabalhar com dados amostrais pode-se utilizar a estatística clássica ou a geoestatística. A escolha entre ambas está relacionada à variabilidade espacial entre as amostras. A estatística clássica baseia-se na hipótese de que não há correlação espacial entre os dados, ou seja, os valores de cada observação são distribuídos aleatoriamente não sendo relacionados à sua localização no espaço (CAMARGO, 1997).

A geoestatística surgiu com Krige (1951) que, enquanto trabalhava com dados de concentração de ouro, verificou que os parâmetros da estatística clássica eram insuficientes para explicar o fenômeno em estudo, e que seria necessário considerar a distância entre as observações. Esse foi o primeiro passo para a utilização da localização geográfica e da dependência espacial para representar um fenômeno em estudo.

No caso do mercado imobiliário estes processos espaciais, principalmente quando se trata da avaliação em massa, estão presentes e causam a autocorrelação espacial. Para Gonzáles (2002) se a variável tem distribuição espacial, as variações nos dados ocorrem em todas as direções. A autocorrelação espacial é geral e está ligada a características peculiares dos 
dados, espacialmente distribuídos, que têm maior relação com os imóveis da vizinhança próxima do que com os mais distantes.

A Geoestatística estuda o comportamento das variáveis regionalizadas, que pode ser feito utilizando o semivariograma e a Krigagem, que são as principais ferramentas dos métodos geoestatísticos (SANTOS, 2014).

\subsection{Semivariograma}

A geoestatística baseia-se nas hipóteses de estacionariedade e média, que pressupõem que as observações mais próximas apresentam um comportamento mais semelhante entre si do que as separadas por uma distância maior. E para verificar a existência da dependência espacial utilizase o semivariograma, que mede o grau de semelhança entre os pares de pontos (CAMARGO, 1997).

O gráfico do semivariograma representa o que se espera dos dados de campo, ou seja, para que haja dependência espacial é necessário que o valor absoluto da diferença entre duas amostras cresça conforme aumenta a distância, até chegar a certo limite onde a partir dali não haja mais influência entre elas (CAMARGO et al 2002).

Na Figura 1 podemos observar os parâmetros do semivariograma, que é representado pelo gráfico da semivariância $\left(\hat{\gamma}_{(h)}\right)$ versus a distância $(h)$.

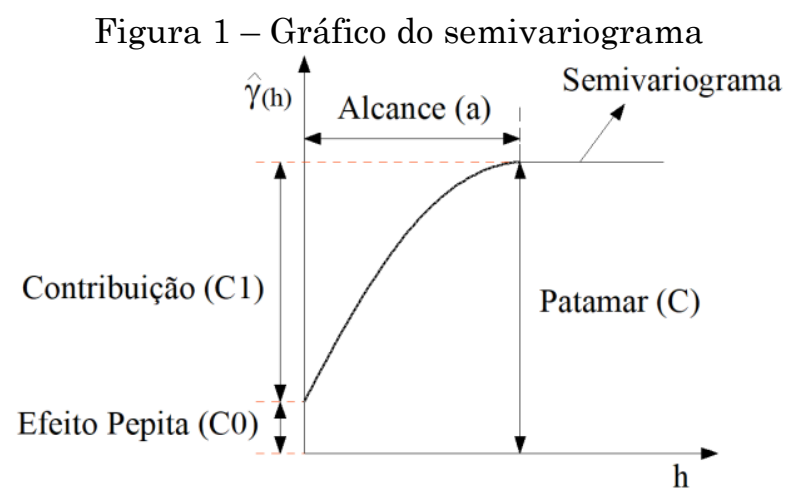

Fonte: Adaptado de CAMARGO (1997). 
Sendo:

- Efeito Pepita (C0): é o valor da semivariância para a distância zero e representa a componente da variabilidade espacial que não pode ser relacionada com uma causa específica, diz respeito a erros aleatórios.

- Contribuição (C1): valor da semivariância após o efeito pepita, na qual ainda há correlação espacial entre as amostras.

- Patamar (C): valor do semivariograma correspondente a seu alcance (a), onde a partir dele não haverá mais dependência espacial entre as amostras, pois a diferença de semivariância para um par de amostras ao longo das distâncias será constante. É o resultado da soma $\mathrm{C} 0+\mathrm{C} 1$.

- Alcance (a): distância na qual ainda há uma correlação espacial entre as amostras.

\subsection{Krigagem}

Comprovada a existência de dependência espacial, através do ajuste do semivariograma, pode-se utilizar a geoestatística para determinar os valores em locais não amostrados, no limite da área de estudo, sem tendência e com variância mínima, através do método conhecido como interpolação por Krigagem (TRIVELLONI, 2005).

A Krigagem se diferencia dos demais métodos de interpolação, pois determina os pesos a partir de uma análise espacial que é baseada no semivariograma experimental. Fora isso, o procedimento é parecido com o de interpolação por média móvel ponderada. Ademais, a Krigagem fornece, em média, estimativas não tendenciosas e com variância mínima (CAMARGO, 1997). Segundo Trivelloni (2005), há três abordagens para a modelagem da variabilidade espacial das amostras, sendo elas:

- Modelos determinísticos de efeitos locais: os pontos da superfície são estimados a partir da interpolação das amostras mais próximas. Nessa abordagem há a suposição de que predominam os efeitos locais. 
- Modelos determinísticos de efeitos globais: nesta abordagem a suposição é de que predominam os efeitos em larga escala e os efeitos locais não são significativos.

- Modelos estatísticos de efeitos locais e globais (Krigagem): os pontos da superfície são estimados a partir da interpolação das amostras mais próximas, utilizando um estimador estatístico.

Segundo Landim (2006), a vantagem da utilização da Krigagem é que a mesma fornece o erro associado aos valores das estimativas, enquanto os demais algoritmos fornecem apenas os valores estimados.

Existem diversos tipos de Krigagem, como a Simples, a Normal, a CoKrigagem, entre outras, sendo a Krigagem ordinária a mais utilizada.

"A Krigagem ordinária é geralmente associada como B.L.U.E. (best linear unbiased estimator). A Krigagem ordinária é 'linear' porque suas estimativas são combinações lineares ponderadas dos dados disponíveis; é 'não-viciada' porque busca o valor de erro ou resíduo médio igual a 0; e é 'melhor' porque minimiza a variância dos erros". (ISAAKS E SRIVASTAVA, 1989, apud JAKOB, 2002).

\section{Planta de Valores Genéricos (PVG)}

A PVG é o resultado da interpolação dos valores estimados dos imóveis, fornecendo assim uma superfície com isolinhas (ou isovalores) onde cada linha representa um valor, sendo possível visualizar a distribuição espacial dos valores dos imóveis da região em estudo.

Conforme a NBR 14653-2 (ABNT, 2011), entende-se por planta de valores a representação gráfica ou listagem dos valores genéricos de metro quadrado de terreno ou do imóvel numa mesma data.

A PVG é um importante instrumento da tributação imobiliária, pois possibilita uma maior justiça fiscal. Mesmo assim, a maioria dos municípios a utiliza apenas em áreas urbanas. Há a necessidade de trabalhos que 
mostrem a importância da aplicação desta ferramenta em mercados de terras rurais (SANTOS, 2014).

Segundo o referido autor, a maioria das PVG dos municípios brasileiros são referidas aos imóveis urbanos, e para os imóveis rurais são utilizadas tabelas de preços por regiões, não existindo técnicas de avaliação preconizadas pela NBR 14653-1 e 3.

A maioria das PVG ainda são elaboradas utilizando métodos de estatística descritiva ou métodos empíricos para calcular os valores dos imóveis, mesmo que os métodos econométricos tornem a avaliação mais objetiva e os resultados menos viesados (MACHADO, 2006).

Uma PVG atualizada permite obter uma base de cálculo para cobrança dos tributos imobiliários de maneira a garantir a equidade fiscal e a prática da justiça tributária, e ainda auxilia no planejamento urbano, entretanto, a maioria das PVG não são atualizadas com frequência (MACHADO, 2006 e SANTOS, 2014). Mas segundo Liporoni (2007), com o advento de técnicas de Geoprocessamento a atualização da PVG ficou mais prática, podendo ser apenas corrigida monetariamente por índices inflacionários, o que possibilita uma atualização mais frequente.

Os modelos de regressão podem ser utilizados para elaborar a PVG, mas após a obtenção do modelo de regressão deve-se utilizar amostras de verificação para validar e garantir a integridade do mesmo (MALAMAN et al. 2012). Além disso, o poder de predição do modelo deve ser verificado a partir do gráfico de preços observados versus valores estimados pelo modelo, o qual deve apresentar pontos próximos à bissetriz do primeiro quadrante (ABNT, 2004). Essa verificação está ilustrada na Figura 2. 
Figura 2 - Gráfico de preços observados versus valores estimados

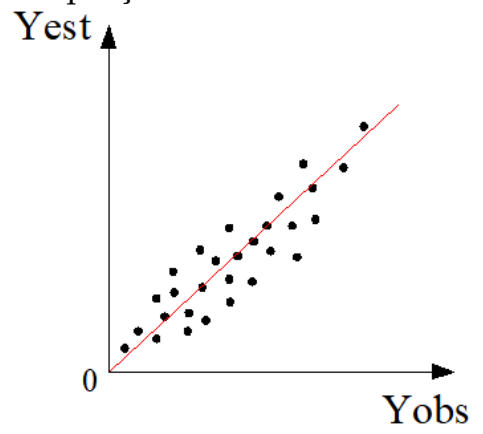

Fonte: Elaborada pelos autores.

\section{Monitoramento do Desempenho Global da Avaliação}

Segundo Erba et al (2005), a qualidade das avaliações irá refletir na distribuição da carga tributária, o que pode ocasionar injustiças fiscais na cobrança dos tributos. Portanto deve haver um maior controle nas avaliações para que os possíveis erros sejam devidamente identificados e eliminados.

Para verificar a integridade das avaliações em massa de imóveis, devemos conferir se as mesmas cumprem as condições da International Association of Assessing Officers (IAAO) para serem consideradas de boa qualidade (TRIVELLONI, 2005).

Segundo Trivelloni (2005), essa análise é realizada comparando os valores obtidos do modelo com os valores reais de mercado, e verificando se a dispersão entre esses valores se enquadra nos limites estabelecidos pela IAAO, de modo que quanto menor for a diferença entre esses valores, melhor será a qualidade da avaliação.

No entanto, as amostras utilizadas nos testes para analisar o desempenho das avaliações devem ser diferentes das que foram empregadas na formação do modelo (ERBA et al., 2005).

A IAAO (2013) recomenda o uso da mediana das razões de avaliação como medida para verificar o desempenho global da avaliação, que é obtida da seguinte forma (Equação 1): 


$$
\mathrm{R}=\frac{P C}{P O}
$$

\footnotetext{
Sendo:

$\mathrm{R}$ a razão de avaliação ou ratio de avaliação;

PC o valor obtido pelo modelo; e

PO o respectivo valor de mercado da observação.
}

Com os valores de $\mathrm{R}$, faz-se a mediana.

Para avaliar a dispersão dos parâmetros a medida mais utilizada é o Coeficiente de Dispersão ou COD, que é uma medida de dispersão horizontal que nos fornece informações sobre a uniformidade da avaliação dos imóveis na área de estudo (TRIVELLONI, 2005).

O COD pode ser calculado da seguinte forma:

1) diminui-se de cada razão de avaliação a mediana de todas as razões;

2) é tomado o valor absoluto das diferenças anteriores;

3) calcula-se a média dos valores absolutos;

4) divide-se a média anterior pela mediana das razões de avaliação;

5) multiplica-se por 100.

Devido ao fato do COD ser afetado por valores extremos das razões de avaliação, faz-se um interquartil dessas razões para definir os valores limites de ratios a considerar na análise e através dele calcula-se um intervalo ampliado, multiplicando o intervalo interquartil por 1,5 ou por 3,0 (DAVIS, 2001 apud TRIVELLONI, 2005).

Para medir a equidade vertical da avaliação é utilizado o índice conhecido como Diferencial Relativo ao Preço ou PRD (sigla de Price Related Differential), que serve para detectar diferenças sistemáticas na forma que são avaliados os imóveis de alto e de baixo valor, verificando se existe regressividade ou progressividade na avaliação (IAAO, 2013). 
A regressividade ocorre quando os imóveis de baixo valor são avaliados como uma porcentagem maior do valor de mercado que os imóveis de alto valor, o contrário disso é a progressividade.

O PRD pode ser obtido dividindo-se a média das razões de avaliação pela sua média ponderada.

A IAAO (2013) recomenda para as medidas apresentadas os valores presentes na Tabela 1 .

Tabela 1- Medidas de qualidade da IAAO (2013)

\begin{tabular}{c|c|c}
\hline Medida & Intervalo & Comentário \\
\hline $\begin{array}{c}\text { Mediana das Razões de } \\
\text { Avaliação }\end{array}$ & 0,90 a 1,10 & Para imóveis Rurais \\
\hline COD & 5 a $20 \%$ & $\begin{array}{c}<0,98 \text { (Progressividade na Avaliação) } \\
>1,03 \text { (Regressividade na Avaliação) }\end{array}$ \\
\hline PRD & 0,98 a 1,03 &
\end{tabular}

Fonte: Elaborada pelos autores.

\section{5 Área de Estudo}

O Projeto de Irrigação e Drenagem da Cana-de-Açúcar (PROJIR) abrange os municípios de São João da Barra, São Francisco de Itabapoana, Campos dos Goytacazes, Cardoso Moreira, Macaé e Quissamã. Situa-se na região nordeste do Estado do Rio de Janeiro, entre as latitudes $21^{\circ} 15^{\prime} 20.90^{\prime \prime S}$ e $22^{\circ} 15^{\prime} 16.46^{\prime \prime} \mathrm{S}$, e longitudes $41^{\circ} 45^{\prime} 31.61^{\prime \prime} \mathrm{W}$ (Figura 3), sendo banhado pelas águas dos rios Paraíba do Sul e Muriaé. 


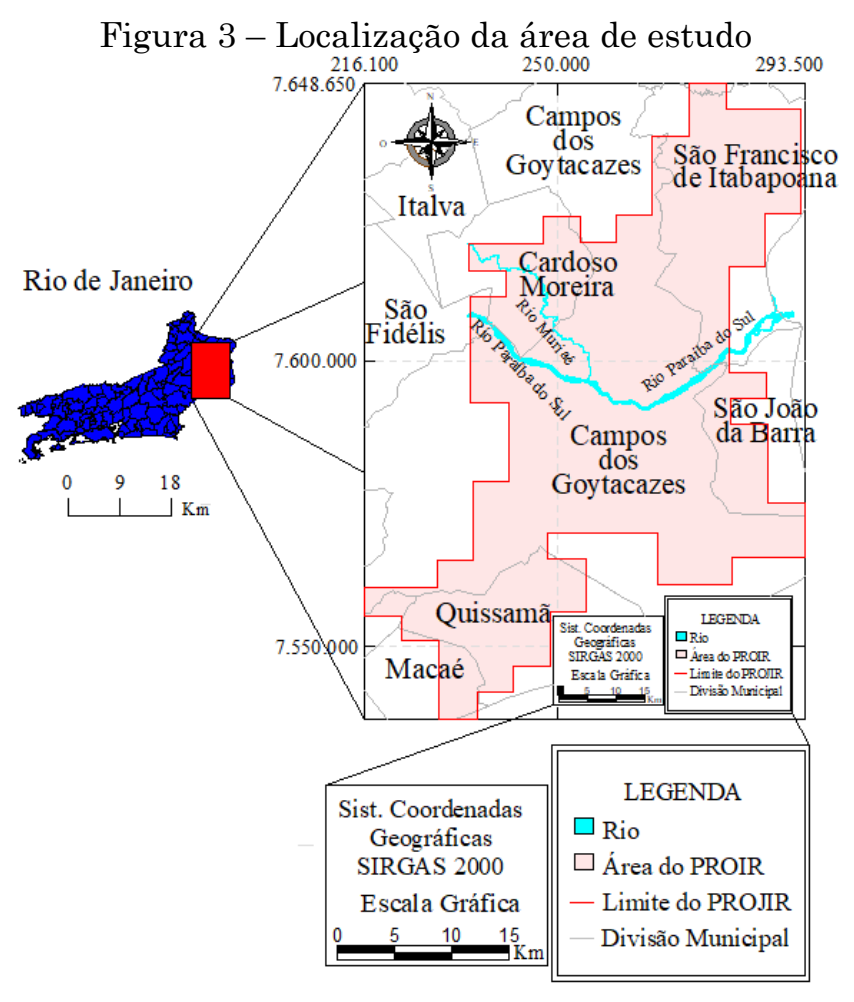

Fonte: Elaborada pelos autores.

O perímetro do PROJIR delimitado para a realização do levantamento é de aproximadamente 250.000,00 ha e correspondia à região de maior concentração da lavoura canavieira.

\section{Materiais}

Para a realização desta pesquisa foram utilizados os seguintes materiais:

- Dados e mapas da área do PROJIR na Região Norte Fluminense RJ, incluindo cartas de cartografia básica e cartas topográficas, de cadastro rural, de classe de solos e de aptidão para irrigação, na escala 1:10.000, e Imagens RapidEye, com resolução de 6,5 metros e fornecidas com espaçamento de pixel de 5 metros;

- Scanner HP Designjet T 2300, com resolução de 600 dpi;

- Softwares: Excel 2010; PCI Geomatica 9.1; SPRING 5.2.3; ArcGIS 10.2; AutoCAD 2007 e Infer 32. 


\section{Método}

7.1 Coleta de dados

Para a realização deste trabalho foram coletadas 138 amostras de imóveis rurais à venda ou transacionados, que corresponde a 1,42\% do total de imóveis, na região do PROJIR, onde 113 amostras foram utilizadas na regressão múltipla e 25 foram utilizadas como amostras de verificação, para validar o modelo encontrado.

\subsection{Escaneamento e Georreferenciamento}

Para localizar as amostras espacialmente, as cartas foram digitalizadas e georreferenciadas no software PCI. Em seguida, as amostras dos imóveis rurais e os tipos de solos foram vetorizados, utilizando-se o software ArcGis, a partir das cartas de cadastro rural e das cartas de aptidão agrícola para irrigação, que se encontravam dentro dos limites das amostras dos imóveis.

7.3 Correção atmosférica e cálculo do NDVI

As imagens RapidEye foram transformadas para reflectância no topo da superfície utilizando um aplicativo de modelo de transferência radiativa para as correções atmosféricas das imagens RapidEye (ANTUNES, DEBIASI e SIQUEIRA, 2014). As imagens foram exportadas para o formato RAW utilizando o software SPRING e após as correções atmosféricas as imagens foram reimportadas para o cálculo do Normalized Difference Vegetation Index (NDVI). Através desse índice foram realizadas as análises sobre a cobertura vegetal da região do PROJIR. 
7.4 Classificação das imagens RapidEye

Após a transformação, as imagens foram classificadas com o objetivo de extrair as informações de interesse, relacionadas às áreas de Preservação Permanente (APP), reflorestamento e mata ciliar, para verificar se a presença dessas áreas contribuiria na valoração dos imóveis na região do PROJIR.

7.5 Obtenção das variáveis independentes

Com todos os materiais disponíveis devidamente tratados, foram coletadas as seguintes variáveis independentes:

Variáveis Quantitativas: coordenadas UTM da localização do imóvel (m), distância a polo de valorização $(\mathrm{km})$, distância ao mar $(\mathrm{km})$, área de mata ciliar (ha), área de Reserva Legal (ha), área de Reflorestamento (ha), frente (m), profundidade (m), área total (ha) e índice de irrigação (\%);

Variáveis Qualitativas Dicotômicas: disponibilidade de água, rios ou córregos, açudes, formato, acesso direto à via principal e cultura;

Variáveis Qualitativas Codificadas: vias de acesso, trafegabilidade, topografia e situação.

No decorrer do projeto houve problemas quanto à aquisição dos perfis de solo para a determinação da capacidade de uso do solo para cada amostra da área de estudo, sendo então utilizado o índice de irrigação como variável que influencia o valor do imóvel. Este índice foi calculado através de uma média ponderada do somatório da produtividade por classe dividida pela área total da amostra.

O primeiro passo para a regressão múltipla ocorreu com a transformação das variáveis qualitativas em quantitativas para que as mesmas pudessem ser utilizadas na regressão. A transformação ocorreu atribuindo valores numéricos aos valores nominais, sendo que os valores mais altos representam a situação favorável e os valores mais baixos a situação desfavorável. 
7.6 Análise Exploratória dos Dados

Para essa análise verificou-se em planilha eletrônica os tipos de transformações que melhor representavam a variável dependente (valor unitário), através de histogramas, box-plot e gráficos Quantil-Quantil.

Verificou-se também se havia casos de correlação linear elevada entre as variáveis independentes, isto é, multicolinearidade. De acordo com a NBR 14653-3 (ABNT, 2004), em caso de multicolinearidade, deve-se examinar a coerência das características do imóvel avaliando com a estrutura de multicolinearidade inferida, e vedar a utilização do modelo em caso de incoerência.

Ainda segundo a NBR 14653-3 (ABNT, 2004), deve-se atentar a valores de correlação linear superiores à 0,8 . Neste estudo, foram considerados como valores críticos os acima de 0,7 .

\subsection{Estimação do Modelo da Regressão}

A regressão múltipla foi utilizada para homogeneizar as diversas características associadas à valoração dos imóveis da região do PROJIR e, desse modo, estimar valores, para serem utilizados na geoestatística, que sejam mais adequados à interpolação dos dados nos locais não amostrados.

Para realizar a regressão foram escolhidas algumas transformações que pudessem representar adequadamente as variáveis independentes, de modo que o modelo da regressão fosse linearizado.

Depois de um processo iterativo, foi encontrado o modelo de regressão que atende aos seguintes pressupostos da NBR 14653-3 (ABNT, 2004): linearidade, normalidade, média dos resíduos igual a zero, homocedasticidade, auto correlação, e ausência de pontos outliers.

Devido ao fato dos valores das propriedades rurais serem relacionados à sua disposição espacial, em conjunto com a regressão múltipla utilizou-se a 
krigagem ordinária para representar o mercado imobiliário da região do PROJIR.

7.8 Geoestatística: Semivariograma e Interpolação dos dados em locais não amostrados

Devido ao fato do mercado imobiliário apresentar o comportamento das variáveis regionalizadas, ou seja, imóveis mais próximos têm valor mais parecido e esse valor tende a se tornar mais discrepante à medida que a distância entre os mesmos aumenta, foi utilizada a geoestatística para estudar o comportamento espacial dos dados estimados pela regressão múltipla e gerar a superfície de valores.

$\mathrm{Na}$ geoestatística foram utilizados os centroides das amostras e seus respectivos valores previstos, obtidos no modelo de regressão.

Para verificar a existência de dependência espacial e escolher o modelo que seria utilizado para ajustar os dados na interpolação, foram feitas comparações entre os principais modelos utilizados na literatura: Esférico, Exponencial e Gaussiano, comparando os parâmetros fornecidos por seus semivariogramas (Efeito Pepita, Patamar, Alcance, Dependência Espacial, Erro Médio Quadrático, entre outros). Escolhido o modelo, e comprovada a existência de dependência espacial, foi elaborada a PVG utilizando-se o Software ArcGIS, e como método de interpolação a Krigagem Ordinária.

\subsection{Análise do Desempenho da Avaliação}

Para analisar o desempenho do modelo de regressão obtido, foram utilizadas as 25 amostras de validação; e através desse modelo foram obtidos os valores estimados para cada uma dessas 25 amostras. Em seguida, os valores observados foram comparados com os valores estimados pelo modelo. Essas comparações foram feitas utilizando medidas como a mediana das 
razões de avaliação, o Diferencial Relativo ao Preço e o Coeficiente de Dispersão.

De posse dos resultados foram comparadas as medidas obtidas com as estabelecidas pela IAAO (2013), verificando assim o desempenho da avaliação.

Como não há uma norma específica para avaliar a superfície de valores para áreas rurais, também utilizamos as métricas da IAAO e as 25 amostras de validação para essa providência.

\section{Resultados e Discussões}

\subsection{Modelo de regressão}

Depois de transformações nas variáveis como transformação logarítmica, potência, raíz, entre outras, e da retirada de alguns pontos outliers, foi encontrado o modelo de regressão que alcançou todos os pressupostos da regressão múltipla, e possui as seguintes variáveis independentes: Vias de acesso (VA), Distância a polo de valorização (DPV), Distância ao mar (DM), Açudes (AÇU), Área Total (AT), Topografia (TOPO), Situação (SIT), Índice de Irrigação (INIRRI) e Cultura (CULT). Na Tabela 2 encontram-se as estatísticas da regressão deste modelo.

Tabela 2 - Disposição das variáveis e estatísticas da regressão múltipla

\begin{tabular}{c|c|c|c}
\hline Variável & Coeficientes & Valor t & P-Valor \\
\hline Interseção & 8,5505 & 39,4097 & 0,0000 \\
\hline VA & 0,0393 & 4,0412 & 0,0001 \\
\hline INV_DPV & 2,2700 & 1,8376 & 0,0694 \\
\hline INV_DM & 2,6720 & 3,0704 & 0,0028 \\
\hline AÇU & 0,0296 & 2,7042 & 0,0082 \\
\hline LN_AT & $-0,0812$ & $-3,4606$ & 0,0008 \\
\hline INV_TOPO & $-0,2271$ & $-2,0953$ & 0,0389 \\
\hline LN_SIT & 0,5288 & 4,6899 & 0,0000 \\
\hline
\end{tabular}




\begin{tabular}{c|c|c|c} 
INV_INIRRI & $-0,0510$ & $-1,6246$ & 0,1077 \\
\hline INV_CULT & 0,3061 & 3,4455 & 0,0009 \\
\hline $\mathbf{R}$ & \multicolumn{3}{|c}{0,7971} \\
\hline $\mathbf{R}^{2}$ & 0,6354 \\
\hline Fcalc & \multicolumn{3}{|c}{17,820} \\
\hline Erro padrão da regressão & \multicolumn{3}{|c}{0,3523} \\
\hline
\end{tabular}

Fonte: Elaborada pelos autores.

\subsection{Interpolação}

Para a construção do semivariograma experimental foi considerado 2,41 km de distância entre os pontos, e no mínimo 52 pares para o cálculo de cada ponto, totalizando 12 pontos.

Para a construção do semivariograma teórico que melhor ajustasse o semivariograma experimental foi utilizado o modelo exponencial, pois foi o que apresentou o menor Erro Médio Quadrático (Root Mean Square - RMS), o maior alcance e a dependência espacial mais forte.

8.3 Análise do Desempenho da Avaliação e elaboração da PVG

As métricas da IAAO permitem verificar quais características do modelo precisam de maior atenção, facilitando assim a construção de modelos mais eficientes.

As diferenças entre o valor previsto pelo modelo da regressão múltipla e o valor observado, das 25 amostras de validação, proporcionaram os resultados da Mediana das Razões de Avaliação, PRD e COD (Tabela 3). 
Tabela 3 - Resultados das medidas da IAAO, referentes à regressão múltipla e à Krigagem, respectivamente

\begin{tabular}{c|c|c}
\hline \multicolumn{3}{c}{ Regressão Múltipla } \\
\hline $\begin{array}{c}\text { Mediana das } \\
\text { Razões de Avaliação }\end{array}$ & PRD & COD \\
\hline 0,98 & & \\
\hline
\end{tabular}

Fonte: Elaborada pelos autores.

No modelo obtido, a principal característica a ser aperfeiçoada é o COD, visto que a mediana das razões de avaliação se enquadra no intervalo estabelecido pela norma e que o PRD está ligeiramente acima do recomendado. Isso provavelmente é devido à extensão da região do PROJIR, que deveria ser dividida em regiões menores, mas não foi possível devido à quantidade de dados disponíveis.

Para a Krigagem (Tabela 4), a mediana das razões de avaliação também se enquadrou no intervalo estabelecido pela IAAO, e tanto o PRD quanto o COD se distanciaram mais do intervalo.

Isso ocorreu, pois, para gerar a superfície de valores, foram utilizados apenas o valor unitário estimado pela regressão e a localização das amostras. Mas foi visto no modelo de regressão que esse valor é controlado tanto pela localização quanto por diversas outras variáveis independentes. Sendo assim, o modelo gerado pela Krigagem terá por base um menor detalhamento de informações, além da falta de amostras e da distância entre as mesmas, fornecendo assim uma precisão menor.

Tabela 4 - Resultados das medidas da IAAO, referentes à regressão múltipla e à Krigagem, respectivamente

\begin{tabular}{ccc}
\multicolumn{3}{c}{ respectivamente } \\
\hline \multicolumn{3}{c}{ Krigagem Ordinária } \\
\hline $\begin{array}{l}\text { Mediana das } \\
\text { Razões de } \\
\text { Avaliação }\end{array}$ & PRD & COD \\
\hline 1,00 & 1,33 & 36,32 \\
\hline Fonte: Elaborada pelos autores.
\end{tabular}


Por fim, foi gerada a PVG final (Figura 4), utilizando a Krigagem Ordinária e o modelo exponencial. Verificou-se na PVG que os valores mais altos estão nos entornos dos centros urbanos das regiões de Campos dos Goytacazes, São Francisco de Itabapoana e Quissamã, e à medida que aumenta a distância a esses centros os valores tendem a diminuir. E os valores também foram maiores à direita devido à proximidade do litoral, e em São João da Barra devido ao Porto do Açu, localizado neste município. 
Figura 4 - Planta de Valores Genéricos final, gerada utilizando a Krigagem Ordinária e o Modelo Exponencial

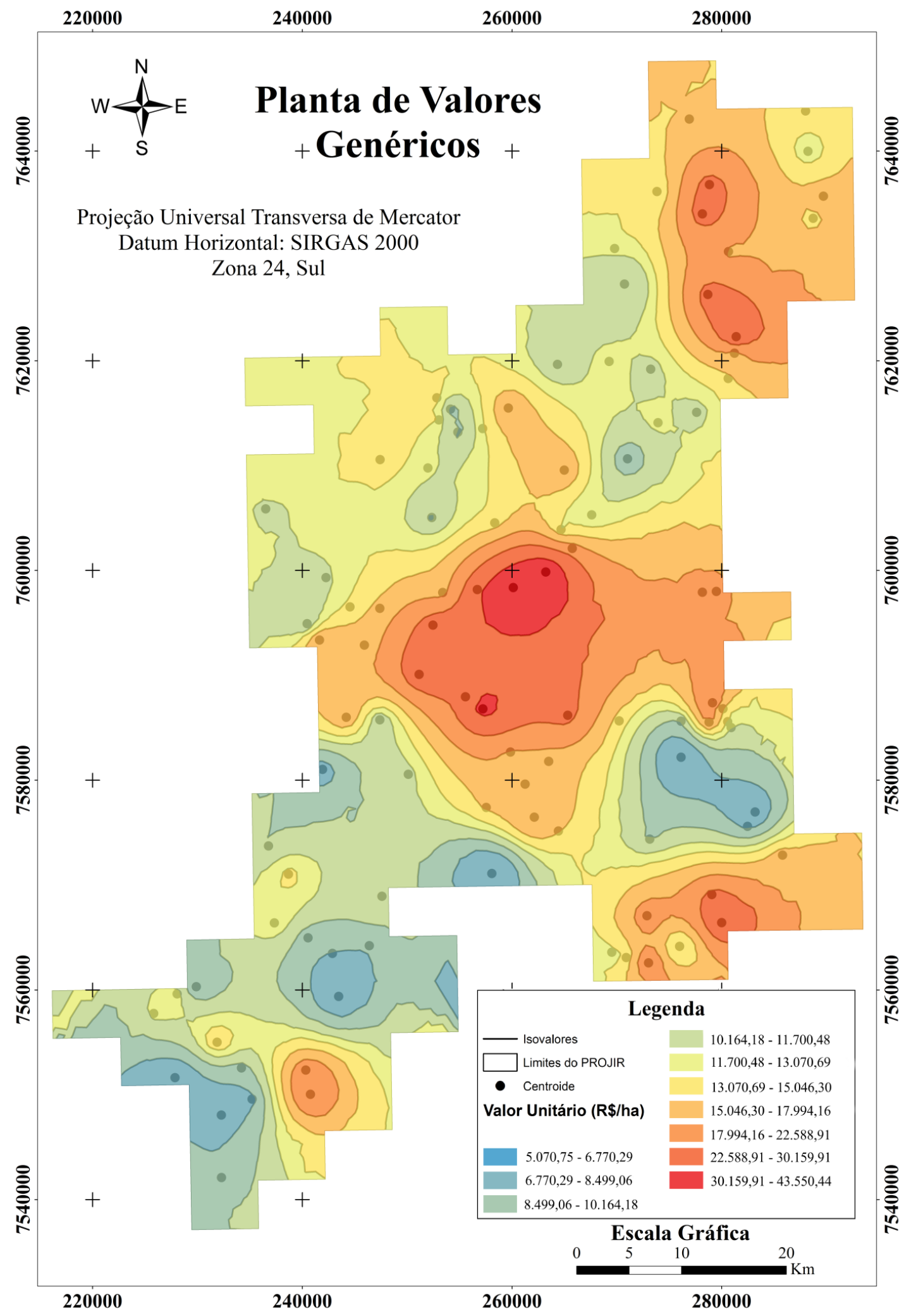

Fonte: Elaborada pelos autores. 


\section{Conclusões}

A utilização da regressão múltipla através das técnicas de inferência estatística permitiu a redução da subjetividade da avaliação e a estimação de um modelo consistente, que alcançou a todos os pressupostos estabelecidos para um modelo de regressão clássico.

O método comparativo direto de dados de mercado permitiu estimar os valores dos imóveis tendo por base os reais valores praticados no mercado imobiliário, o que tornou a avaliação mais objetiva e as informações estimadas por ela mais confiáveis e íntegras.

As amostras de validação mostraram-se de fundamental importância na verificação da qualidade do ajustamento do modelo e da interpolação de dados em locais não amostrados.

A metodologia proposta, mediante a utilização da regressão múltipla em conjunto com a geoestatística, mostrou-se eficiente na elaboração da superfície de valores, pois representou de forma satisfatória os valores dos imóveis rurais na região do PROJIR.

A utilização das normas internacionais para analisar o desempenho da avaliação proporcionou um discernimento sobre o ajustamento dos modelos encontrados e indicou que a Krigagem perde um pouco do refinamento da regressão múltipla. Na regressão múltipla foram utilizadas as variáveis formadoras do valor (variáveis relacionadas às características dos imóveis), enquanto na Krigagem a superfície de valores foi definida em função da dependência espacial dos valores unitários dos imóveis. O menor detalhamento de parâmetros na utilização da Krigagem gerou aumento dos valores das métricas da IAAO (mais especificamente no COD), sendo necessária uma quantidade maior de amostras para alcançar os intervalos estipulados pela norma.

No entanto, os resultados mostram que a utilização da regressão múltipla em conjunto com a geoestatística, na avaliação em massa de imóveis 
rurais, é viável e realça a importância de se ter uma quantidade de amostras compatível com a área de estudo.

Visto que o ITR é um importante instrumento de justiça fiscal e que há uma grande escassez de estudos sobre a valoração cadastral rural, estudos como este são fundamentais à comunidade científica e para a sociedade no geral.

\section{Agradecimentos}

Registra-se aqui o nosso agradecimento ao PIBIC/CNPq pela concessão da bolsa de Iniciação Científica à primeira autora, número de processo 118151/2015-0.

\section{Contribuição dos autores}

O presente trabalho apresenta os resultados do PIBIC da primeira autora. E foi parte da tese de doutorado da Profa. Dra. Marlene Salete Uberti. O Prof. Dr. Mauro Antonio Homem Antunes foi o orientador da primeira autora. E a Profa. Dra. Paula Debiasi foi co-orientadora do doutorado da Profa. Dra. Marlene Salete Uberti, e colaboradora neste projeto. Os três professores participaram das revisões do artigo.

\section{Referências}

ANTUNES, M. A. H.; DEBIASI, P.; SIQUEIRA, J. C. S. Avaliação espectral e geométrica das imagens RapidEye e seu potencial para o mapeamento e monitoramento agrícola e ambiental. Revista Brasileira de Cartografia, vol. 66, n. 1, 2014. pp. 105-113.

ABNT - ASSOCIAÇÃO BRASILEIRA DE NORMAS TÉCNICAS. Avaliações de Bens Parte 2: Imóveis Urbanos. NBR 14653-1. Rio de Janeiro. ABNT. 2011. 
ABNT - ASSOCIAÇÃO BRASILEIRA DE NORMAS TÉCNICAS. Avaliação de Bens Parte 3: Imóveis Rurais. NBR 14653-3. Rio de Janeiro. ABNT. 2004.

BRASIL. Constituição da República Federativa do Brasil. Brasília. Diário Oficial da União. 1988. 292 p.

BRASIL. Lei n ${ }^{\circ} 9.393$ de 19 de dezembro de 1996. Imposto Territorial Rural. Brasília. Diário Oficial da União, 22 janeiro de 1997.

BRASIL. Lei n 10.267 de 6 de abril de 1966. Brasília. Diário Oficial da União, 28 de agosto de 2001.

BRASIL. Lei $\mathrm{n}^{\circ} 11.250$ de 27 de dezembro de 2005. Brasília. Diário Oficial da União. 27 de dezembro de 2005.

BRASIL, Secretaria da Receita Federal - SRF. Análise da Arrecadação das Receitas Federais. Dezembro de 2012. Disponível em: <http://www.receita.fazenda.gov.br/publico/arre/2012/Analisemensaldez12.pdf $>$ Acesso em: 09/2015.

CÂMARA, E. C. G; FUCKS, S. D; CÂMARA, G. Análise espacial de superfícies. FUCKS, S. D. et al. Análise espacial de dados geográficos. $1^{a}$ ed. São José dos Campos: $\quad 2002.2$ Disponível em: $<$ http://www.dpi.inpe.br/gilberto/tutoriais/analise/cap3.pdf $>$ Acesso em: 05/2019.

CAMARGO, E.C.G. Desenvolvimento, Implementação e Teste de Procedimentos Geoestatísticos (Krigagem) no Sistema de Processamento de Informações Georreferenciadas (SPRING). Dissertação de Mestrado. Instituto Nacional de Pesquisas Espaciais. Programa de Pós-Graduação em Sensoriamento Remoto. São José dos Campos. 1997. $123 p$.

DAVIS, P. The IAAO Standard on Ratio Studies: a Framework for Order and Progress in Assessment Performance Evaluation. Anais do Seminário Internacional sobre Tributação Imobiliária, Porto Alegre, 2001.

ERBA, D. A. DE OLIVEIRA, F. L; JUNIOR, P. N. L. Cadastro Multifinalitário Como Instrumento da Política Fiscal e Urbana. Rio de Janeiro. Organizador. 2005. 144p.

GONZÁleZ, M. A. S. Aplicação de Técnicas de Descobrimento de Conhecimento em Base de Dados e de Inteligência Artificial em 
Avaliações de Imóveis. Tese de Doutorado. Universidade Federal do Rio Grande do Sul, Programa de Pós-Graduação em Engenharia Civil, Porto Alegre, 2002. 300p.

IAAO - INTERNATIONAL ASSOCIATION OF ASSESSING OFFICERS. Standards on Mass Appraisal of Property. Missouri: IAAO, 2013.

JAKOB, A. A. E. A krigagem como método de análise de dados demográficos. XIII

Encontro da Associação Brasileira de Estudos Populacionais, Ouro Preto, 2002. pp. 1-21. Disponível em:<http://www.abep.nepo.unicamp.br/docs/anais/pdf/2002/GT_SAU_ST3_Ja kob_texto.pdf $>$. Acesso em: 11/2015.

KRIGE, D. G. A statistical approch to some basic mine evaluation problems on the Witwatersrand. Johanesburg Chemistry Metallurgy Mining Society South African, vol. 52, n 6, 1951. pp. 119-139. Disponível em: $<$ http://www.saimm.co.za/Conferences/DanieKrige/DGK01b.pdf>. Acesso em $12 / 2017$.

LANDIM P.M.B. 2006. Sobre Geoestatística e mapas. TerræDidatica, vol. 2, n. 1, 2007. $\quad$ pp. 19-33. Disponível em:<http://www.fca.unesp.br/Home/Instituicao/Departamentos/CienciadoSolo/ gepag/t_didatica_2006_v02n01_p019-033_landim.pdf>. Acesso em: 11/2015.

LIPORONI, A. S. Avaliações em massa com ênfase em planta de valores. IBAPE-SP. ed. 1. Engenharia de Avaliações. Editora Pini, 2007. pp. 957-982.

MACHADO, E. F. Elaboração de Planta de Valores Genéricos para zona rural com uso de Superfície de Tendência. Dissertação de Mestrado. Universidade Federal de Santa Catarina, Programa de Pós-Graduação em Engenharia Civil, Florianópolis, 2006. 105 p.

MALAMAN, C. S.; MARQUES, A. P. S.; TACHIBANA, V. M.; AMORIM, A. Elaboração de planta de valores genéricos a partir de distintos modelos de regressão linear múltipla. Anais do IV Simpósio Brasileiro de Ciências Geodésicas e Tecnologias da Geoinformação, Recife, 2012. pp. 9.

NETO, A. M. Imposto Territorial Rural (ITR): algumas considerações. Indicadores Econômicos FEE, vol. 20, n. 3, 1992. pp. 185-199,

OLIVEIRA, T. A. M. Imposto Territorial Rural: um Estudo Econômico sobre a Descentralização da Cobrança. Tese de Doutorado. Universidade 
Estadual de Campinas, Curso de Pós-Graduação em Desenvolvimento Econômico, Campinas, 2010. 135 p.

REYDON, B. P.; OLIVEIRA, T. A. M. A Descentralização Fiscal do ITR Através do

Cadastro Territorial Multifinalitário. Anais do IV Simpósio Brasileiro de Ciências Geodésicas e Tecnologias da Geoinformação. Recife, 2012. pp. 001-007.

SANTOS, H. G. Efeitos Espaciais Em Mercados De Terras Rurais: Modelagem, Validação E Avaliação De Desempenho. Dissertação de Mestrado. Universidade Federal de Pernambuco, Programa de Pós-graduação em Ciências Geodésicas e Tecnologias da Geoinformação, Recife, 2014. 182 p.

TRIVELLONI, C. A. P. Método Para Determinação do Valor da Localização Com Uso de Técnicas Inferenciais e Geoestatísticas na Avaliação em Massa de Imóveis. Tese de Doutorado. Universidade Federal de Santa Catarina, Programa de Pós-Graduação em Engenharia Civil, Florianópolis, 2005. 172p.

UBERTI, M. S. Avaliação em Massa de Imóveis Rurais Através de Modelagem Clássica, Espacial e Geoestatística. Tese de Doutorado. Universidade Federal Rural do Rio de Janeiro, Pró-Reitoria de Pesquisa e Pós-Graduação, Seropédica, 2016. 129p.

VILARINHO, C.R.O. O imposto territorial rural (ITR) no Brasil. Dissertação de Mestrado. Universidade Estadual de Campinas, Curso de Pós-graduação em Economia, Campinas, 1989. 258p. 